\title{
ON TAUBERIAN THEOREMS FOR ABEL-CESÀRO SUMMABILITY
}

\author{
by C. T. RAJAGOPAL \\ (Received 1lth January, 1958)
}

1. Introduction. For a series $\sum a_{n}$ with partial sums $A_{n}=a_{0}+a_{1}+\ldots+a_{n}(n \geqslant 0)$, supposed to be real in this note, we define, in a generally accepted notation ([2], pp. 7, 9, 94-98), the following transforms :

(C, $\alpha)$ sequence-transform, $\alpha>-1: C_{n}^{\alpha}=\frac{1}{E_{n}^{\alpha}} \sum_{\nu=0}^{n} A_{\nu} E_{n-\nu}^{\alpha-1}, E_{n}^{\alpha}=\frac{\Gamma(n+\alpha+1)}{\Gamma(\alpha+1) \Gamma(n+1)}(\alpha \neq 0)$,

$$
C_{n}^{0}=A_{n},
$$

$(\mathrm{H}, k)$ sequence-transform $(k=0,1,2, \ldots): H_{n}^{k}=\frac{1}{n+1} \sum_{\nu=0}^{n} H_{\nu}^{k-1}(k \geqslant 1), H_{n}^{0}=A_{n}$

$(\mathrm{A} ; \mathrm{C}, \alpha)$ function-transform, $\alpha>-1: f_{\alpha}(x) \equiv f_{\alpha}\left(x ; A_{\nu}\right)=(1-x) \sum_{\nu=0}^{\infty} C_{\nu}^{\alpha} x^{n}(0 \leqslant x<1)$.

We also define as follows the corresponding summabilities of the series $\Sigma a_{n}$ or the sequence $A_{n}(n \geqslant 0)$, each to a value $l$ understood to be always finite in this note.

(C, $\alpha$ ) summability to $l: C_{n}^{\alpha} \rightarrow l$ as $n \rightarrow \infty$,

$(\mathrm{H}, k)$ summability to $l: H_{n}^{k} \rightarrow l$ as $n \rightarrow \infty$,

(A; C, $\alpha$ ) summability to $l: f_{\alpha}(x) \rightarrow l$ as $x \rightarrow 1-0$.

The summability last defined, which we shall call Abel-Cesàro summability, reduces to Abel or (A) summability when $\alpha=0$.

(A; C, $\alpha$ ) summability, with the restriction $\alpha \geqslant 0$, seems to have been considered explicitly first by Kogbetliantz (e.g. [6], p. 37) and later by Lord ([7], §§ 1-3). The former writer denotes our $f_{\alpha}(x)$ by $x^{-\alpha} f^{(\alpha)}(x)$ while the latter defines $f_{\alpha}(x)$ as here but refers to our summability (A; $\mathrm{C}, \alpha)$ as summability $(C, \alpha ; A)$. In a recent paper [1], Amir (Jakimovski) has studied summability $(\mathrm{A} ; \mathrm{C}, \alpha)$ for all $\alpha>-1$, without reference to the earlier writers mentioned above, using the less explicit notation $(A, \alpha)$ summability instead of $(\mathrm{A} ; \mathrm{C}, \alpha)$ summability; and his main theorems overlap in part those of the earlier writers.* For instance, his consistency theorem ([1], Theorem 2.1 or Theorem 2.2), that $(\mathrm{A} ; \mathrm{C}, \alpha)$ summability for an $\alpha>-1$ implies (A; C, $\beta$ ) summability for any $\beta>\alpha$, is given by Lord ([7], Lemma 7) in a more general form for $\alpha \geqslant 0$, and his inclusion theorem ([1], Theorem 2.3), that $(\mathrm{C}, \beta)$ summability for a $\beta>-1$ implies $(\mathrm{A} ; \mathrm{C}, \alpha)$ summability for any $\alpha>-1$, appears in the more general form of Lemma 2 of $\S 2$ in Lord's paper [7]. As regards his Tauberian theorems, what appears to be new in them ([1], Theorem 3.1, Theorem 3.2 and Corollary) may be separated from what is essentially not ([1], Theorem 2.4 and Corollary, Theorem 3.3) and embodied in a single statement as under.

Theorem A. If a sequence $A_{n}(n \geqslant 0)$ is summable $(\mathrm{A} ; \mathrm{C}, \beta)$ to $l$ for some $\beta>-1$ and there is an $\alpha \geqslant \beta$ such that

* This fact is not 'mentioned in the notices of Jakimovski's paper [1] in Math. Reviews, 13 (1952), 835, and Zbl. für Math., 47 (1953), 86. 
$\lim _{\delta \rightarrow+0} \liminf _{n \rightarrow \infty} \min _{n \leqslant m \leqslant(1+\delta) n}\left(C_{m}^{\alpha}-C_{n}^{\alpha}\right) \geqslant 0$,

then $A_{n}$ is summable $(\mathrm{C}, \alpha)$ to $l$.

In the case $\beta=0$, we can have $\alpha$ satisfying either $\alpha \geqslant \beta=0$ or $0=\beta>\alpha>-1$.

It is the main object of this note to prove a result, Theorem $B$, which extends Theorem $A$ by dispensing with the restriction $\alpha \geqslant \beta$ even when $\beta \neq 0$, i.e. by assuming only $\alpha>-1$. The proof given of Theorem $B$ is manifestly different from and simpler than the proof of Theorem A. It uses the symbolic operators $\mathrm{C}^{(\alpha)}$ and $\mathrm{H}^{k}$ for the transforms $(\mathrm{C}, \alpha)$ and $(\mathrm{H}, k)$ in much the same way as the latter operator has been used by me [11] to simplify the proofs of some theorems of Jakimovski, and by Parameswaran and Jakimovski [8] to generalise the said theorems.

2. Lemmas. The lemmas required in the proof of Theorem $B$ are presented below, independently of the classical case $\alpha=\beta=0$ of the theorem. For the first lemma I am indebted to the referee who, however, points out that, although the lemma does not seem to have been explicitly stated before, it is not essentially new as its proof is exactly like that of its particular case $p=0$ given by Hausdorff ([3], III) and independently by Szász ([12], pp. 1243-5, §3).

Lemma 1. Let $A_{n}(n \geqslant 0)$ be a sequence of which any regular Hausdorff transform is the sequence $\mathfrak{G}\left[A_{n}\right] . \quad$ Let $f(x) \equiv f_{0}(x), g(x)$ be the Abel transforms of $A_{n}, \mathfrak{G}\left[A_{n}\right]$ respectively. Let $p \geqslant 0$. Then

implies

$$
f(x)=o\left\{(1-x)^{-p}\right\} \text { as } x \rightarrow 1-0,
$$

$$
g(x)=o\left\{(1-x)^{-p}\right\} \text { as } x \rightarrow 1-0 .
$$

In the case $p>0$, the result is true more generally for a conservative Hausdorff transformation $\mathfrak{5}$.

Proof. It may be recalled that the proof in the case $p=0$ follows readily from the relation ([12], p. 1244, last line)

$$
g(x)=\int_{0}^{1} f\left(\frac{t x}{1-x+t x}\right) d \psi(t)
$$

where $\psi(t)$ is the function of bounded variation in $0 \leqslant t \leqslant 1$ generating the moment sequence $\mu_{n}(n \geqslant 0)$ which defines the transformation $\mathcal{G}$ as usual ([2], pp. 249, 256).

To prove the more general result in the case $p>0$, we note that

$$
0 \leqslant \frac{t x}{1-x+t x} \leqslant x \text { for } 0 \leqslant t \leqslant 1,
$$

whence it follows that, if $f(x)=o\left\{(1-x)^{-p}\right\}$ with $p>0$, then, corresponding to any given $\epsilon>0$ and $x$ sufficiently near 1 , we have

$$
\left|f\left(\frac{t x}{1-x+t x}\right)\right|<\epsilon(1-x)^{-p} \text { for } 0 \leqslant t \leqslant 1
$$

This gives the result.

Of the next two lemmas, the first is given by Lord ([7], Lemma 7) with $\alpha \geqslant 0$ instead of $\alpha>-1$ and the second as it stands ([7], Lemma 5).

LEMMA $1^{\prime}$. If $p \geqslant 0, \beta>\alpha>-1$, then

$$
f_{\alpha}(x)=o\left\{(1-x)^{-p}\right\} \text { implies } f_{\beta}(x)=o\left\{(1-x)^{-p}\right\} \text { as } x \rightarrow 1-0 \text {. }
$$


Proof. We have to show that

$$
f_{0}\left(x ; C_{\nu}^{\alpha}\right)=o\left\{(1-x)^{-p}\right\} \text { implies } f_{0}\left(x ; C_{v}^{\beta}\right)=o\left\{(1-x)^{-p}\right\} \text { as } x \rightarrow 1-0,
$$

and this result follows from Lemma 1 since $C_{n}^{\beta}$ is a regular Hausdorff transform of $C_{n}^{\alpha}$.

LEMma 2. If $p \geqslant 0, \beta>-1, \alpha>-1$, then

$$
C_{n}^{\beta}=o\left(n^{p}\right) \text { as } n \rightarrow \infty \text {, implies } f_{\alpha}(x)=o\left\{(1-x)^{-p}\right\} \text { as } x \rightarrow 1-0 .
$$

Lemma 3. Let $\mathrm{C}^{(\alpha)}(\alpha>-1)$ and $\mathrm{H}^{k}(k=0,1,2, \ldots)$ denote the operators which when applied to the sequence $A_{n}(n \geqslant 0)$ result in $C_{n}^{\alpha}$ and $H_{n}^{k}$ respectively, so that we can write

Then we have the operational identity

$$
\mathrm{C}^{(\alpha)}\left[A_{n}\right]=C_{n}^{\alpha}, \mathrm{H}^{k}\left[A_{n}\right]=H_{n}^{k} \text {. }
$$

$$
\mathrm{H}^{k} \mathrm{C}^{(\alpha)}=\sum_{r=0}^{k} c_{k, r} \mathrm{H}^{r} \mathrm{C}^{(\alpha+k)} \text {, where } \sum_{r=0}^{k} c_{k, r}=1 \text {. }
$$

To prove (1), which is stated explicitly by Hardy in the case $\alpha=0$ ([2], p. 107, relation (5.11.1) of $\S 5.11$ ), we use induction on $k$, starting from the easily verified case $k=1$ of (1) which is

$$
\mathrm{H}^{1} \mathrm{C}^{(\alpha)}=c_{1,0} \mathrm{C}^{(\alpha+1)}+c_{1,1} \mathrm{H}^{1} \mathrm{C}^{(\alpha+1)}, \quad \text { where } c_{1,0}=\frac{1}{\alpha+1}, c_{1,1}=\frac{\alpha}{\alpha+1} .
$$

LEMMA 4. If a sequence $A_{n}$ is summable $(\mathrm{A} ; \mathrm{C}, \alpha+k)$ to $l$, then the sequence $\mathrm{H}^{k} \mathrm{C}^{(\alpha)}\left[A_{n}\right]$ is summable (A) to $l$.

By Lemma 1 , each of the sequences $\mathrm{H}^{r} \mathrm{C}^{(\alpha+k)}\left[A_{n}\right] \quad(r=0,1,2, \ldots)$, is summable (A) to $l$, and so Lemma 4 follows from (1).

Lemma 5. If a sequence $A_{n}$ is summable (A) to $l$ and satisfies the condition

$$
\left(\mathrm{H}^{0}-\mathrm{H}^{1}\right)\left[A_{n}\right]=O_{L}(\mathbf{1})
$$

then it is summable $(\mathrm{H}, 1)$ to $l$.

Lemma 5 is due to Szász ([12], p. 560, Satz II and p. 626, Theorem II). A short proof of the lemma, in accord with the proof of Theorem $B$, is as follows. The sequence $\left(\mathrm{H}^{0}-\mathrm{H}^{1}\right)\left[A_{n}\right]$ being summable $(\mathrm{A})$ to 0 by Lemma 1 and also bounded on one side, is summable $(\mathrm{H}, 1)$ to 0 by a well-known theorem ([2], p. 154, Theorem 94), i.e.

$$
\mathrm{H}^{1}\left(\mathrm{H}^{0}-\mathrm{H}^{\mathbf{1}}\right)\left[A_{n}\right]=\left(\mathrm{H}^{0}-\mathrm{H}^{1}\right) \mathbf{H}^{1}\left[A_{n}\right] \rightarrow 0 .
$$

From this and the summability (A) to $l$ of $\mathrm{H}^{1}\left[A_{n}\right]$, the desired conclusion that $\mathrm{H}^{1}\left[A_{n}\right] \rightarrow l$ emerges on an appeal to Tauber's second theorem ([2], p. 150, Theorem 86).

Lemma 6. A sequence $A_{n}$ satisfying the condition

$$
\min _{n \leqslant m \leqslant(1+\delta) n}\left(A_{m}-A_{n}\right) \geqslant-K,
$$

for some constants $\delta>0, K>0$, also satisfies the condition (2).

Lemma 6 has been proved by Szász for functions instead of sequences ([12], p. 586, relation (22') of Satz a). However, a proof is inserted here for convenience. If

$$
A_{n}=a_{0}+a_{1}+\ldots+a_{n}
$$

in (3) we get, taking $m=n+1, a_{n+1}=O_{L}(1)$, so that there is a constant $K^{\prime}$ such that

$$
\min _{n \leqslant m \leqslant(1+8) n}\left(a_{n}+a_{n+1}+\ldots+a_{m}\right) \geqslant-K^{\prime} .
$$


Hence, by Abel's lemma,

$$
\begin{gathered}
\sum_{r=1}^{n} r a_{r}=\sum_{k=0}^{\infty} \sum_{(1+\delta)^{-k-1} n<+\leqslant(1+\delta)^{-k} k_{n}}^{r a_{r}} \\
\quad \geqslant \sum_{k=0}^{\infty}(1+\delta)^{-k} n \min _{(1+\delta)^{-k-1} n<N \leqslant M \leqslant(1+\delta)^{-k} k_{n}} \sum_{r=N}^{M} a_{r} \\
\geqslant-K^{\prime} n \sum_{k=0}^{\infty}(1+\delta)^{-k}=-\frac{1+\delta}{\delta} K^{\prime} n, \\
\text { i.e. } \quad A_{n}-\frac{A_{0}+A_{1}+\ldots+A_{n}}{n+1} \geqslant-\frac{1+\delta}{\delta} K^{\prime} \frac{n}{n+1}>-\frac{1+\delta}{\delta} K^{\prime} .
\end{gathered}
$$

Lemma 7. If $A_{n}(n \geqslant 0)$ is summable $(\mathrm{H}, k)$ to $l$ and satisfies the condition

$$
\lim _{\delta \rightarrow+0} \liminf _{n \rightarrow \infty} \min _{n \leqslant m \leqslant(1+\delta) n}\left(A_{m}-A_{n}\right) \geqslant 0 \text {, }
$$

then $A_{n}$ converges to $l$.

In the present context it seems best to follow Knopp ([5], Theorem II) to prove Lemma 7 , by defining the $(\mathrm{H}, k)$ transform for a series $\Sigma a_{n}$ instead of its sequence of partial sums $A_{n}$, for $k=1,2,3, \ldots$ in succession, as another series :

$$
\sum_{0}^{\infty} a_{n}^{(k)} \equiv a_{0}^{(k)}+\sum_{n=1}^{\infty} \frac{a_{1}^{(k-1)}+2 a_{2}^{(k-1)}+\ldots+n a_{n}^{(k-1)}}{n(n+1)}
$$

where

$$
a_{0}^{(k)}=a_{0} \quad(k \geqslant 1), \quad a_{n}^{(0)}=a_{n} \quad(n \geqslant 1),
$$

80 that we have the alternative definition for

$$
(\mathrm{H}, k) \text { summability to } l: \sum_{0}^{\infty} a_{n}^{(k)}=l, \text { where } a_{0}^{(k)}=a_{0} \quad(k=0,1,2, \ldots) .
$$

Now condition (4), in conjunction with Lemma 6, shows that $n a_{n}^{(1)}=O_{L}(1)$ and hence also that $n a_{n}^{(k)}=O_{L}(1)$ for $k=2,3, \ldots$ successively. Since $n a_{n}=O_{L}(1)$ is a special form of condition (4), we thus see that our hypothesis that the partial sums of $\Sigma a_{n}$ satisfy (4) implies that the partial sums of $\Sigma a_{n}^{(k)}(k \geqslant 1)$ again satisfy (4). Consequently, assuming Lemma 7 in the well-known case $k=1([2]$, p. 125, Theorem 68), we prove the lemma for any given $k \geqslant 2$ by noting that its two assumptions, namely $\Sigma a_{n}^{(k)}=l$ and (4), together imply $\Sigma a_{n}^{(r)}=l$ for $r=k-1, k-2, \ldots, 0$ successively.

3. Theorems. Our main Tauberian theorem for Abel-Cesàro summability can now be stated and proved.

Theorem B. If a sequence $A_{n}(n \geqslant 0)$ is summable $(\mathrm{A} ; \mathrm{C}, \beta)$ to $l$ for some $\beta>-1$ and there is an $\alpha>-1$ for which

$$
\lim _{\delta \rightarrow+0} \liminf _{n \rightarrow \infty} \min _{n \leqslant m \leqslant(1+\delta) n}\left(C_{m}^{\alpha}-C_{n}^{\alpha}\right) \geqslant 0,
$$

then $A_{n}$ is summable $(\mathrm{C}, \alpha)$ to $l$.

Proof. Let $k$ be a positive integer such that $\alpha+k \geqslant \beta$ and therefore, by virtue of Lemma $\mathrm{l}^{\prime}, A_{n}$ is summable (A; $\mathrm{C}, \alpha+k$ ) to $l$. Then, by Lemma $4, \mathrm{H}^{k} \mathrm{C}^{(\alpha)}\left[A_{n}\right]$ is summable (A) to $l$. 
On the other hand, by an application of Lemma 6 to (5), we have

$$
C_{n}^{\alpha}-\mathrm{H}^{1}\left[C_{n}^{\alpha}\right]=O_{L}(1),
$$

so that

$$
\mathrm{H}^{k}\left[C_{n}^{\alpha}\right]-\mathrm{H}^{1}\left[\mathrm{H}^{k}\left[C_{n}^{\alpha}\right]\right]=O_{L}(1) .
$$

Lemma 5 , with $\mathrm{H}^{k}\left[C_{n}^{\alpha}\right]$ instead of $A_{n}$, now shows that $\mathrm{H}^{k}\left[C_{n}^{\alpha}\right]$ is summable $(\mathrm{H}, 1)$ to $l$, or $C_{n}^{\alpha}$ is summable $(\mathrm{H}, k+1)$ to $l$; and the conclusion sought follows from an application of Lemma 7 to $C_{n}^{\alpha}$.

After Theorem B, the remaining Tauberian theorems for Abel-Cesàro summability may be included in a single enunciation as follows.

Theorem C. If $p \geqslant 0, \alpha>-1, \beta>-1$ and a sequence $A_{n}(n \geqslant 0)$ satisfies the conditions

$$
\begin{array}{r}
\lim _{\delta \rightarrow+0} \liminf _{n \rightarrow \infty} \min _{n \leqslant m \leqslant(1+\delta) n} \frac{C_{m}^{\alpha}-C_{n}^{\alpha}}{n^{p}} \geqslant 0, \\
f_{\beta}(x)=o\left\{(1-x)^{-p}\right\} \text { as } x \rightarrow 1-0, \ldots \ldots . . \\
C_{n}^{\alpha}=o\left(n^{p}\right) \text { as } n \rightarrow \infty .
\end{array}
$$

then

Proof. The case $p=0$ is disposed of by Theorem B and the case $p>0$ is separately dealt with as briefly indicated below.

It is known (e.g. [4], p. 36, or [12], p. 617, relation (17) of Hilfssatz 1) that, when $p>0$, (7) implies

Hence we have, for $\gamma=\max (\alpha, \beta)$,

$$
C_{n}^{\alpha}=O_{L}\left(n^{p}\right) *
$$

$$
C_{n}^{\nu}=O_{L}\left(n^{p}\right)
$$

and we also know, by Lemma 1 , that (8) holds with $\beta$ replaced by any $\delta \geqslant \beta$ and so with $\delta=\gamma$. Therefore, by a well-known theorem of Hardy and Littlewood ([7], Theorem A, or [4], Satz B with $L(x) \equiv 1$ ),

$$
\mathrm{H}^{1}\left[C_{n}^{\gamma}\right]=o\left(n^{p}\right) \text {. }
$$

If $\gamma=\alpha$, a simple Tauberian argument (e.g. [4], proof of Satz C), involving (7) and (10), establishes our conclusion (9). On the other hand, if $\gamma=\beta,(10)$ can be written in the form

$$
\frac{1}{\gamma+1} C_{n}^{\gamma+1}+\frac{\gamma}{\gamma+1} \mathrm{H}^{1}\left[C_{n}^{\gamma+1}\right]=o\left(n^{p}\right)
$$

and a classical Mercerian argument ([2], p. 104, proof of Theorem 5l) shows that $C_{n}^{\gamma+1}=o\left(n^{p}\right)$. From this we see, by Lemma 2, that (8) holds with $\beta$ replaced by any $\delta>-1$ and so with $\delta=\alpha$. Conclusion (9) now follows from a repetition of our previous argument in the case $\gamma=\alpha$.

Two Tauberian theorems for Abel-Cesaro summability, proved by Lord ([7], Theorems 1,2 ), can now be stated as corollaries, even with his condition $\beta \geqslant 0$ (in the notation adopted here) relaxed to $\beta>-1$.

Corollary $\mathrm{C}_{1}$. Theorem $\mathrm{C}$ can be restated with hypothesis $(7)$ changed to $C_{n}^{\alpha}=O_{L}\left(n^{p}\right)$ and conclusion (9) changed to $C_{n}^{\alpha+1}=o\left(n^{p}\right)$.

* Tbe distinction between the cases $p>0$ and $p=0$ may be explained thus. The implication of (7), - stated above for $p>0$, assumes the different form of (6) for $p=0$. 
The proof is contained in that of Theorem $\mathrm{C}$.

Corollary $\mathrm{C}_{2}$. Theorem $\mathrm{C}$ can be restated with only (7) changed to

$$
C_{n}^{\prime \alpha}=O_{L}\left(n^{p}\right)
$$

where $\mathrm{C}_{n}^{\prime \alpha}$ is the $n$-th $(\mathrm{C}, \alpha)$ mean of the sequence $A_{n}^{\prime} \equiv n a_{n}$.

To deduce Corollary $\mathrm{C}_{2}$ from Theorem $\mathrm{C}$ we have only to observe that ( $7^{\prime}$ ) implies (7). In the case $\alpha=0,\left(7^{\prime}\right)$ becomes $n a_{n}=O_{L}\left(n^{p}\right)$, i.e. $a_{n}=O_{L}\left(n^{p-1}\right)$, from which (7) with $\alpha=0$ readily follows by summation. If $\alpha \neq 0$ but $\alpha>-1,\left(7^{\prime}\right)$ gives

$$
C_{n}^{\alpha}-C_{n-1}^{\alpha}=\frac{\alpha}{n}\left(C_{n}^{\alpha-1}-C_{n}^{\alpha}\right)=\frac{C_{n}^{\prime \alpha}}{n}=O_{L}\left(n^{p-1}\right),
$$

and therefore (7) follows again by summation.

Corollary $\mathrm{C}_{1}$ and Corollary $\mathrm{C}_{2}$, both for $p=0, \alpha \geqslant 0$, are given by Kogbetliantz in a slightly different form ([6], pp. 39, 40, Theorems XIX, XXI). Elsewhere are functional analogues of Corollary $\mathrm{C}_{2}$ in the case $p=0, \alpha \geqslant 1$, involving either the Laplace transform ([9], Corollary II'.1), or more generally, a certain class of integral transforms ([10], Theorem A).

My thanks are due to the referee for his replacement of my original condition $\beta \geqslant 0$ by the condition $\beta>-1$ in Theorem $\mathrm{C}$ and its corollaries, by means of his Lemma 1 .

\section{REFERENCES}

1. A. Amir (Jakimovski), On a converse of Abel's theorem, Proc. Amer. Math. Soc., 3 (1952), 244-256.

2. G. H. Hardy, Divergent series (Oxford, 1949).

3. F. Hausdorff, Die Äquivalenz der Hölderschen und Cesàroschen Grenzwerte negativer Ordnung, Math. Z., 31 (1930), 180-196.

4. J. Karamata, Neuer Beweis und Verallgemeinerung der Tauberschen Sätze, welche die Laplacesche und Stieltjessche Transformation betreffen, J. Reine Angew. Math., 164 (1931), 27-39.

5. K. Knopp, On the proof of the main Teuberian theorem for the $C_{k^{-}}$and $H_{k}$-methods, Proc. Amer. Math. Soc., 5 (1954), 571-573.

6. E. Kogbetliantz, Sommation des séries et intégrales divergentes par les moyennes arithemétiques et typiques, Mémor Sci. Math. LI (Paris, 1931).

7. R. D. Lord, On some relations between the Abel, Borel and Cesàro methods of summation, Proc. London Math. Soc. (2), 38 (1935), 241-256.

8. M. R. Parameswaran and A. Jakimovski, Generalized Tauberian theorems for summability (A), Quart. J. Math. Oxford (2). To appear shortly.

9. C. T. Rajagopal and A. Jakimovski, Applications of a theorem of O. Szász for the product of Cesàro and Laplace transforms, Proc. Amer. Math. Soc., 5 (1954), 370-384.

10. C. T. Rajagopal, Theorems on the product of summability methods with applications, $J$. Indian Math. Soc. (N.S.), 18 (1954), 89-105.

11. C. T. Rajagopal, Simplified proofs of some Tauberian theorems of Jakimovski, Pacific $J$. Math., 7 (1957), 955-960, with addendum and corrigendum, ibid.

12. O. Szász, Collected mathematical papers (Cincinnati, 1955).

Ramanujan Institute of Mathematics

MadRAS

INDIA 